\title{
Emergency gastrectomy for gastric necrosis 5 years after laparoscopic adjustable gastric banding (LAGB)
}

\author{
Rani Kanthan ${ }^{1}$, Jenna-Lynn Senger ${ }^{1}$, Selliah Chandra Kanthan ${ }^{2}$ \\ ${ }^{1}$ Department of Pathology and Laboratory Medicine, University of Saskatchewan, Saskatoon, Canada; \\ ${ }^{2}$ Department of Surgery, University of Saskatchewan, Saskatoon, Canada. \\ Email: rani.kanthan@saskatoonhealthregion.ca
}

Received 27 June 2011; revised 3 August 2011; accepted 10 August 2011.

\begin{abstract}
Laparoscopic adjustable gastric banding (LAGB) is currently one of the most common bariatric surgical procedures performed worldwide for the treatment of morbid obesity. Among bariatric surgeries, the percentage of LAGB has increased from $24.4 \%$ to 42.3\%. In North America alone, the number of LAGB procedures has increased exponentially by $944.2 \%$ (from 9,270 to 96, 800 cases). Though early and late complications following LAGB are well-understood, data regarding long term complications remains indefinite due to the limited follow-up periods. We report a case of acute gastric necrosis associated with band slippage presenting as a surgical emergency five years after LAGB. Gastric necrosis represents a rare, but life threatening complication of gastric banding that has been reported in the early post-operative period. To the best of our knowledge, this case report is the longest time-interval reported between LAGB and the diagnosis of this very rare complication. Accurate diagnosis is often delayed due to its rarity and nonspecific clinical presentation. Increased awareness of this delayed complication should facilitate early recognition as it often requires urgent surgical intervention to prevent fatal outcomes.
\end{abstract}

Keywords: LAGB; Gastric Necrosis; Band Slippage

\section{INTRODUCTION}

Since its introduction in 1993, worldwide, more than 100,000 laparoscopic adjustable gastric banding (LAGB) procedures have been performed as the surgical treatment of choice for morbid obesity [1]. Features including the a) ease of the procedure, b) minimally invasive nature, c) the potential reversibility, and d) the ability to calibrate the stoma contribute to the continued growing popularity of this technique [2]. Early or late complica- tions related to LAGB include band slippage, pouch dilatation, band erosion, gastric perforation, injectionport malfunction and rarely gastric necrosis [3]. Though, specific late complications associated with this procedure are recognized in $10 \%-20 \%$ of cases [1] the data remains incomplete due to limited follow-up periods.

Gastric necrosis is a very rare complication that is potentially life threatening and constitutes an absolute surgical emergency with dire consequences if unrecognized. Including this report, a total of eighteen cases have been reported in the literature (Table 1) [1-16].

We report the case of a patient who, five years after LAGB, presented with vague abdominal symptoms to the Emergency Room (ER) on two occasions prior to being accurately diagnosed as gastric pouch necrosis which necessitated urgent surgical intervention.

\section{CASE REPORT}

A 46-year-old woman presented to our emergency department with progressive abdominal pain, abdominal distension, nausea and vomiting. Abdominal examination showed mild tenderness in the epigastrium on palpation with no other positive signs. She had been to the ER on two previous occasions in the past few weeks for similar complaints. Relevant past history included a laparoscopic adjustable gastric band placement in Mexico five years ago for weight reduction (225 lbs). Due to the repeated visits and the persistence of symptoms over 24 hours a surgical consult with radiological investigations were undertaken.

Plain films of the abdomen confirmed the presence of a LAGB attached to its external port as seen in Figure 1. A contrast enhanced abdominal CT scan revealed markedly dilated esophagus (Figure 2a) and stomach with a distal migration of the LAG band (Figure 2b) at the pylorus (Figure 2c). An abnormal enhancement pattern with early 'target sign' of alternating layers of high and low attenuation suggestive of submucosal edema and/or hemorrhage in keeping with mucosal ischemia is de- 


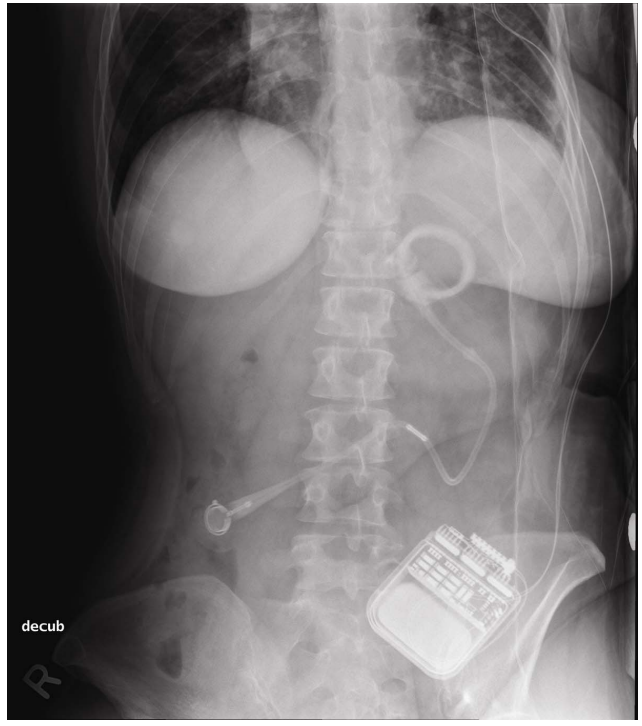

Figure 1. Plain $x$-ray abdomen demonstrates the presence of adjustable gastric band (AGB) and port.
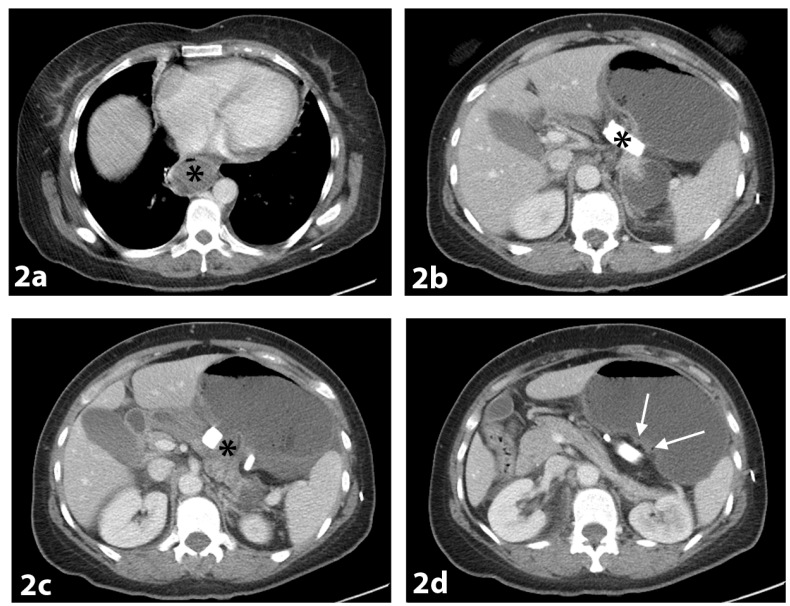

Figure 2. Contrast enhanced helical-axial images-CT scan of the abdomen demonstrating a) marked distension of the esophagus $(*)$ posterior to the heart, b) LAGB $(*)$ seen in the distal portion of the dilated stomach, c) LAGB position confirmed to be at the pylorus $(*)$ and d) early 'target sign' (arrow $\downarrow$ ) with alternating layers of high and low attenuation suggestive of submucosal edema and/or hemorrhage in keeping with mucosal ischemia is identified.

monstrated in Figure 2d. The CT scan confirmed gastric outlet obstruction with query migration/slippage of the LAGB and the suggestion of intramural gas raised the strong suspicion of imminent gastric ischemia. The position of the gastric band appeared to have changed in orientation and was found to be located more distally than expected.

The patient was taken to the operating room for emergency exploratory laparoscopy, which revealed an adherent, abnormal-appearing stomach with vascular compromise. It was decided to proceed with an open laparotomy that showed the gastric band had slipped to the pylorus, causing ischemia of the entire stomach above the band up to the gastroesophageal junction. The gastric band was removed. As there was no reversal of ischemia despite intra-operative warming aids, a total gastrectomy was performed, with an esophagojejunostomy, Roux-en-Y reconstruction and the creation of a feeding jejunostomy.

Pathological examination of the stomach showed dark blackened nonviable mucosa of the entire stomach with pink viable proximal and distal resection margins at the esophagus and the duodenum respectively (Figure $\mathbf{3 a}$ ). Histological examination of stomach sections proximal to the band revealed full-thickness ischemic necrosis with excessive hemorrhage, extensive submucosal edema, dilated vessels, inflammatory infiltrate and edema of the muscularis layer (Figures $\mathbf{3 b}$ and $\mathbf{c}$ ). Distal to the band, microscopic sections continued to show regions of interrupted focal mucosal necrosis with surviving islands of viable mucosa (Figure 3d). Post-operatively the patient was discharged on jejunostomy tube and oral feeds.

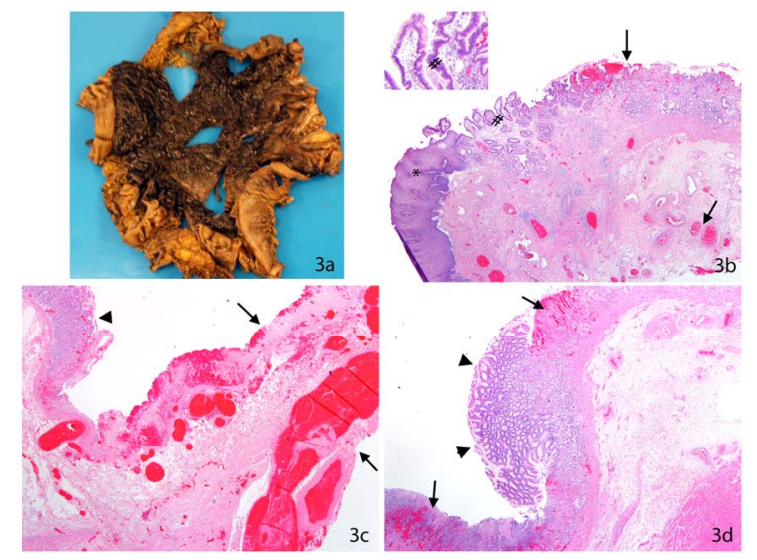

Figure 3. a, Open gastrectomy specimen demonstrating ischemic non-viable mucosa in the center with viable margins at the periphery; b, Haematoxylin and eosin stained slide of the gastroesophageal junction (magnification objective lens x2) showing viable squamous-lined esophagus $\left(^{*}\right)$ adjacent to columnar-lined GE junction (\#) with evidence of acute ischemic changes in the adjacent mucosa with congestion, submucosal edema, and dilatation of vessels (arrow $\downarrow$ ). An insert image in the top left-hand corner (\#) is a high-power magnification of the adjacent columnar-lined epithelium with no evidence of metaplasia.; c, Haematoxylin and eosin stained slide (magnification objective lens x2) showing full-thickness ischemic necrosis of the stomach (arrow $\downarrow$ ) adjacent to focal partialthickness ischemia (arrowhead $\mathbf{\Delta}$ ); d, Haematoxylin and eosin stained slide (magnification objective lens x2) showing 'mucosal islands' (arrowhead $\mathbf{\Delta}$ ) amidst ongoing ischemic regions (arrow $\downarrow$ ). 
One week later she returned to the ER complaining of abdominal pain with cramping, depression and a general sense of being overwhelmed. She was admitted with the suspicion of a partial small bowel obstruction; however, abdominal CT scan, HIDA scan and gastroscopy all failed to demonstrate the same. She was started on TPN to give bowel rest, and upon re-starting oral feeds did well. She was followed up in the clinic 6 weeks postoperatively and is progressing well. Her current weight is $121 \mathrm{lbs}$.

\section{REVIEW AND DISCUSSION}

Using PubMed, a literature search was performed using the text words 'laparoscopic gastric band*' and 'gastric necrosis'. Overall, 27 results including case reports and case series were retrieved. All papers relevant to this study were reviewed and of these, 22 papers have been included in this study. The findings of the cases with gastric necrosis following LAGB including details of : a) reference \#, b) author, c) study type, d) number of cases/patients reported, e) age and sex, f) time interval between LAGB and gastric necrosis, g) presenting symptoms, h) management, i) outcome and j) cause of the gastric necrosis are summarized in Table 1 [1-16].

Since its introduction in 1993 and approval by the Foods and Drug Administration in 2001, Laparoscopic Adjustable Gastric Banding (LAGB) has gained popularity for the treatment of morbid obesity, making it one of the most common surgeries in Europe, Australia, Latin America, and the USA [4,5]. The ease of this surgical technique coupled with the minimal invasiveness and its reversibility has contributed to its increasing popularity over the past five years [2]. Worldwide, while the percentage of LAGB carried out among bariatric surgeries has increased from $24.4 \%$ in 2003 to $42.3 \%$; in North America alone in 2008 the number of LAGB procedures has increased exponentially by $944.2 \%$ (from 9,270 to 96,800 cases) [17].

It is estimated that band related complications arise in $12.2 \%$ of LAGB cases $[5,18]$. These can occur as early or late postoperative events. Band related complications include band migration/erosion, band dislocation/ leakage, band slippage/prolapse and port related leaks/rupture [19]. The most common complication is band slippage or gastric prolapse occurring in $4.5 \%$ - $5 \%$ of patients [5,18]. Additional complications may include bleeding, gastric perforation, pouch or esophageal dilatation, stoma obstruction, band erosion and gastric necrosis [5,8]. Rarely, small bowel obstruction secondary to intragastric erosion and migration of a gastric band has also been reported [20]. Clinical presenting symptoms of these late complications usually include gradual onset of reflux, dysphagia, and vomiting [2]; however, acute retrosternal chest pain has also been reported as the primary presenting complaint [6]. In many cases, band deflation relieves symptoms [1]. When this is insufficient, laparoscopic treatment, including band repositioning,replacement, or removal is an adequate remedy for many of these complications. Though specific late complications associated with this procedure are recognized in $10 \%-20 \%$ of cases [1] the data remains indefinite due to limited follow-up periods. Gastric necrosis is a very rare complication that is potentially life threatening and constitutes an absolute surgical emergency.

As seen in Table 1, several mechanisms as the cause of gastric necrosis were identified by individual authors in their respective reports. In agreement with the literature, gastric prolapse was the most commonly reported cause of necrosis [21]. Additional mechanisms reported as the cause of necrosis include band slippage [22], fundal herniation [1], pouch dilatation [16], late prolapse [10], Type II paraesophageal hernia with strangulation [6] paragastric Richter's hernia [22] and organo-axial volvulus [11]. Though many complications arising from LAGB occur during the immediate or early post-operative phase of healing, gastric necrosis appears to be a late delayed complication, with the majority of cases occurring 1-3 years after LAGB (Table 1). If the necrosis is detected early, conservative treatment with complete deflation of the adjustable band with close follow-up [7], or limited gastrectomy procedures such as sleeve gastrectomy [8] have been reported as effective treatment. In the event of complete gastric necrosis, a total gastrectomy is often required. Therefore, early detection of gastric necrosis is crucial to satisfactory patient management. The reported data of follow-up periods following LAGB are generally less than five years [4]. As gastric necrosis can occur as a late delayed complication (after five years) as seen in this reported case, a more vigilant long term follow-up of LAGB cases is recommended.

The stomach is a highly vascular organ with an extensive arcade of arterial anastomotic network which therefore rarely succumbs to ischemic necrosis [3]. In this context, for ischemia to occur, several predisposing risk factors have been identified to contribute to the onset of gastric devitalization. These include:

A) Gastric prolapse occurs where a part of the stomach slips through the band. This can decrease the blood flow within the gastric pouch. This band slippage may occur immediately after surgery or many months later, in either an anterior or posterior direction which causes an obstruction between the upper and lower stomach [21].

B) Pouch dilatation increases the pressure on the gastric wall, impairing the vasculature [8]. This can be caused by patients who chronically overeat, and will 
Table 1. Composite literature review of gastric necrosis following LAGB (pubmed listed 2001-2011) in descending chronological order.

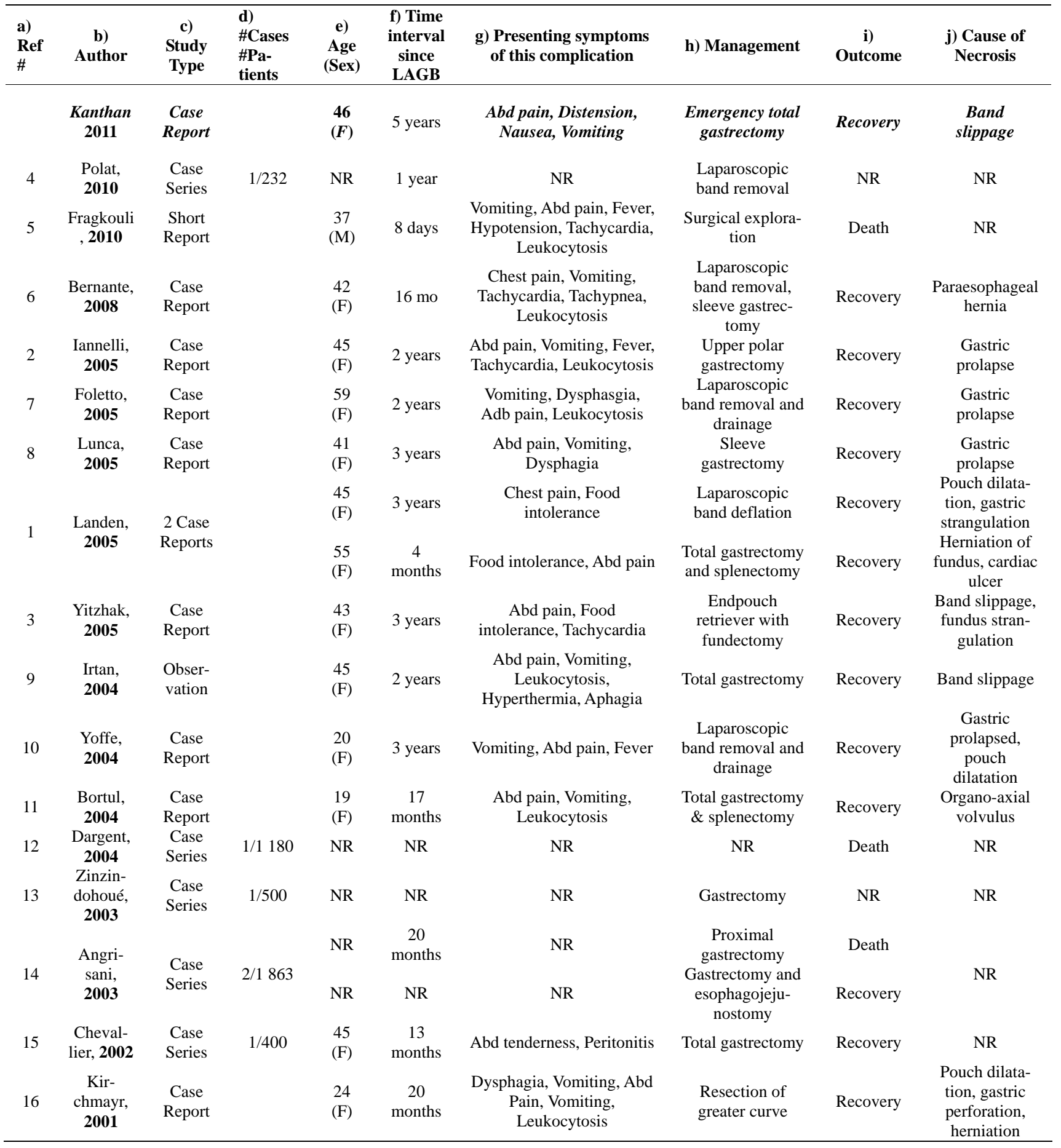

often present with vomiting caused by stoma obstruction and gastroesophageal junction motility disorders [16,21].

C) Band slippage/migration/erosion may result in the exertion of pressure against the fundus, limiting blood flow to the gastric wall $[6,19]$. The risk of a slipped band increases dramatically in patients who self-vomit after meals and in those who ingest large amounts of food [7].

D) Despite the rich arterial anastomoses in the stomach that can prevent necrosis, pre-existing co-morbidities found among obese people may further increase the risk of gastric necrosis [5]. 
E) Finally, over-inflation of the gastric band can cause the same increased pressure, limiting blood flow. No more than $0.5 \mathrm{cc}$ should be injected per visit and checked monthly [10].

Due to its rarity, the recognition and accurate diagnosis of gastric necrosis is often delayed; therefore, its presentation often constitutes a medical emergency necessitating urgent surgical intervention. As illustrated by our index case, gastric necrosis can present as a delayed complication five years after LAGB. Though delayed gastric necrosis has been reported earlier as listed in Table 1, this case has the longest post procedural time interval (5 years). Failure to recognize this urgent clinical situation can have dire consequences which, if untreated, will result in death of the patient. [5]. While conservative treatment has been described in some cases of focal limited early necrosis, most cases require surgical intervention with procedures ranging from minimally invasive resection $[3,16]$ including emergency sleeve gastrectomy [6] to emergency total gastrectomy $[1,9,14$, $15]$.

In conclusion, as obesity rates rise to epidemic proportions, it is likely that LAGB will continue to escalate in popularity as the preferred method of surgical treatment. Awareness and recognition of complications that may arise immediately or years following LAGB is important data to be collected for continued best practice care of these patients. Delayed occurrence of gastric necrosis represents a rare but life threatening complication of gastric banding. Accurate diagnosis is often delayed due to its rarity and nonspecific clinical signs at presentation. Increased awareness of this delayed complication should facilitate early recognition as it will often require urgent surgical intervention to avert dire consequences.

\section{REFERENCES}

[1] Landen, S., Majerus, B. and Delugeau, V. (2005) Complications of gastric banding presenting to the ED. American Journal of Emergency Medicine, 23, 368-370. doi:10.1016/j.ajem.2005.02.016

[2] Iannelli, A., Facchiano, E., Sejor, E., et al. (2005) Gastric necrosis: A rare complication of gastric banding. Obesity Surgery, 15, 1211-1214. doi:10.1381/0960892055002194

[3] Yitzhak, A., Avinoach, E. and Mizrahi, S. (2005) A minimally invasive solution for necrotic fundus following slipped adjustable gastric band. Obesity Surgery, 15, 439-441. doi:10.1381/0960892053576631

[4] Polat, F., Poyck, P.P.C., Dickhoff, C., et al. (2010) Outcome of 232 morbidly obese patients treated with laparoscopic adjustable gastric banding between 1995-2003. Digestive Surgery, 27, 397-402. doi:10.1159/000318778

[5] Fragkouli, K., Mitselou, A. and Vougiouklakis, T. (2010) Death-related gastric necrosis after laparoscopic adjustable gastric banding in the early post-operative period.
Diagnostic Pathology, 5, 68. doi:10.1186/1746-1596-5-68

[6] Bernante, P., Breda, C., Zangrandi, F., et al. (2008) Emergency sleeve gastrectomy as a rescue treatment for acute gastric necrosis due to type II paraesophageal hernia in an obese woman with gastric banding. Obesity Surgery, 18, 737-741. doi:10.1007/s11695-007-9374-x

[7] Foletto, M., De Marchi, F., Bernante, P., et al. (2005) Late gastric pouch necrosis after Lap-Band treated by an individualized conservative approach. Obesity Surgery, 15, 1487-1490. doi:10.1381/0960892057748859272

[8] Lunca, S., Vix, M., Rikkers, A., et al. (2005) Late gastric prolapse with pouch necrosis after laparoscopic adjustable gastric banding. Obesity Surgery, 15, 571-575. doi:10.1381/0960892053723420

[9] Irtan, S., Forestier, D., Scesa, J.L., et al. (2004) Nécrose gastrique après gastroplastie. La Presse Médicale, 33, 718-720. doi:10.1016/S0755-4982(04)98728-3

[10] Yoffe, B., Sapojnikov, S. and Goldblum, C. (2004) Gastric wall necrosis following late prolapse after laparoscopic banding. Obesity Surgery, 14, 142-144. doi:10.1381/096089204772787473

[11] Bortul, M., Scaramucci, M., Tonello, C., et al. (2004) Gastric wall necrosis from organo-axial volvulus as a late complication of laparoscopic gastric banding. Obesity Surgery, 14, 285-287. doi:10.1381/096089204322857726

[12] Dargent, J. (2004) Surgical treatment of morbid obesity by adjustable gastric band: the case for a conservative strategy in the case of failure-a 9-year series. Obesity Surgery, 14, 986-990. doi:10.1381/0960892041719545

[13] Zinzindohoué, F., Chevallier, J.M., Douard, R., et al. (2003) Laparoscopic gastric banding: a minimally invasive surgical treatment for morbid obesity: Prospective study of 500 consecutive patients. Annals of Surgery, 237, 1-9. doi:10.1097/00000658-200301000-00001

[14] Angrisani, L., Furbetta, F., Doldi, S.B., et al. (2003) Lap Band adjustable gastric banding system: The Italian experience with 1863 patients operated on 6 years. Surgical Endoscopy, 17, 409-412.

[15] Chevallier, J.M., Zinzindohoué, F., Elian, N., et al. (2002) Adjustable gastric banding in a public university hospital: prospective analysis of 400 patients. Obesity Surgery, 12, 93-99. doi:10.1381/096089202321144658

[16] Kirchmayr, W., Ammann, K., Aigner, F., et al. (2001) Pouch dilatation after gastric banding causing gastric necrosis. Obesity Surgery, 11, 770-772. doi:10.1381/09608920160558768

[17] Buchwald, H. and Oien, D.M. (2009) Metabolic/bariatric surgery worldwide 2008. Obesity Surgery, 19, 16051611. doi:10.1007/s11695-009-0014-5

[18] Carelli, A.M., Youn, H.A., Kurian, M.S. et al. (2010) Safety of the laparoscopic adjustable gastric band: 7-year data from a U.S. center of excellence. Surgical Endoscopy, 24, 1819-1823. doi:10.1007/s00464-009-0858-8

[19] Forsell, P., Hallerbäck, B., Glise, H. et al. (1999) Complications following Swedish Adjustable Gastric Banding: a long-term follow-up. Obesity Surgery, 9, 11-16. doi:10.1381/096089299765553647

[20] Egbeare, D.M., Myers, A.F. and Lawrance, R.J. (2008) Small bowel obstruction secondary to intragastric erosion and migration of a gastric band. Journal of Gastrointestinal Surgery, 12, 983-984. 
doi:10.1007/s11605-007-0304-y

[21] Spivak H and Favretti F. (2002) Avoiding postoperative complications with the LAP-BAND system. The American Journal of Surgery, 184, 31S-37S.

doi:10.1016/S0002-9610(02)01177-7

[22] Srikanth, M.S., Oh, K.H., Keskey, T., et al. (2005) Criti- cal extreme anterior slippage (paragastric Richter's hernia) of the stomach after laparoscopic adjustable gastric banding: Early recognition and prevention of gastric strangulation. Obesity Surgery, 15, 207-215. doi:10.1381/0960892053268471 